ITP-SB-95-21

hep-th/9507014

July 3, 1995

\title{
Non-Abelian Axial-Vector Duality: \\ a Geometric Description
}

\author{
Eugene Tyurin \\ Institute for Theoretical Physics, SUNY at Stony Brook, \\ Stony Brook, NY 11794-3840, USA \\ E-mail: gene@insti.physics.sunysb.edu
}

\begin{abstract}
We give a geometric characterization of the quasi axial-vector (KiritsisObers) target space duality in the spirit of the bi-algebra (KlimčikŠevera) approach. We show that the $\sigma$-models constructed by taking quotients have non-abelian chiral currents that obey "non-commutative conservation laws" and provide the criterion for a $\sigma$-model to have a dual using the axial-vector procedure.
\end{abstract}




\section{Introduction}

The field of non-abelian target space duality is the next, more complicated step towards a possible understanding of the global structure of the space of string vacua and, in particular, compactification and cosmological issues.

The original form of non-abelian duality [3, 4] started with a $\sigma$-model that was acted on by a group of non-abelian isometries; this led to the puzzle of the "vanishing isometries": the inability to perform an inverse duality transformation, because the model obtained did not have the isometries of the original $\sigma$-model.

Besides [3, 4], there were several more attempts to develop a different approach to non-abelian duality: works by Sfetsos [5], Álvarez, Álvarez-Gaumé and Lozano [9, 10], Kiritsis and Obers [2], and, the most encouraging one, by Klimčik and Severa [1].

In the abelian case, there are two equivalent ways to obtain dual models: the method of Lagrange multipliers [8] and the method of axial-vector quotients [7]. It turns out that these two methods yield mutually incompatible results when applied to the $\sigma$-models with non-abelian isometries.

Recently, Klimčik and Ševera [1] suggested a way to give up one of the central concepts of T-duality: the need for an isometry of the target space manifold. Their generalized non-abelian duality (GNAD) is built around the bi-algebra of quasi-isometries (as opposed to the usual path-integral manipulations) and it includes the "traditional" 3, 4, non-abelian duality as a special case.

In this letter we analyze non-abelian axial-vector duality (NAAVD) [2] and find that it produces a family (parametrized by the group of external automorphisms of the isometry group) of dual models that admit "noncommutative conservation laws" [四]. They do not form a bi-algebra, but by using them, we have been able to give the Kiritsis-Obers duality a geometric characterization: a condition that has to be satisfied by a $\sigma$-model in order to have a dual model. Such condition replaces the need for the existence of Killing vectors; and it is different from the one needed for the Klimčik-Ševera duality.

It seems highly unlikely that these two approaches can be reconciled, though this has not been rigorously proven yet. This suggests that the unified

\footnotetext{
${ }^{1}$ There is also a Hamiltonian approach to the T-duality (for a review see e.g. [9]), but it is beyond the scope of this paper.
} 
picture of target space dualities may be even more complex than conjectured in [1].

The outline of the paper is as follows. First, we briefly describe abelian axial-vector duality $[7,6$. 6 . Next, we summarize the core notions of the GNAD [1]. In Section 1 we study the axial-vector duality of non-abelian isometries 22 and show how it relates to the ideas of GNAD. The conclusions and the discussion are presented in the Section 5 .

\section{Abelian Duality and Quotients}

We start with the most general $\sigma$-model action that admits abelian (anti)chiral currents:

$$
\begin{aligned}
\mathcal{S}_{D+2}\left[\theta_{i}, x\right]= & -\frac{1}{2} \int d^{2} \sigma\left[\partial \theta_{1} \bar{\partial} \theta_{1}+\partial \theta_{2} \bar{\partial} \theta_{2}+2 B(x) \partial \theta_{2} \bar{\partial} \theta_{1}\right. \\
& \left.+F_{i 1}(x) \partial x^{i} \bar{\partial} \theta_{1}+F_{2 j}(x) \partial \theta_{2} \bar{\partial} x^{j}\right]+\mathcal{S}_{\mathcal{X}}[x, \boldsymbol{\Phi}] \\
\mathcal{S}_{\mathcal{X}}= & -\frac{1}{2} \int d^{2} \sigma\left[F_{i j}(x) \partial x^{i} \bar{\partial} x^{j}-\frac{\sqrt{\gamma}}{4} \mathbf{R}^{(2)} \boldsymbol{\Phi}(x)\right]
\end{aligned}
$$

where the conserved currents are given by

$$
J=\partial \theta_{1}+B \partial \theta_{2}+\frac{1}{2} F_{i 1} \partial x^{i} \quad \bar{J}=\bar{\partial} \theta_{2}+B \bar{\partial} \theta_{1}+\frac{1}{2} F_{2 j} \bar{\partial} x^{j}
$$

We can take a quotient with respect to the axial-vector symmetry of (2.1) using the minimal coupling $(\varepsilon= \pm 1)$

$$
\partial_{ \pm} \theta_{1} \longrightarrow \partial_{ \pm} \theta_{1}+A_{ \pm} \quad \partial_{ \pm} \theta_{2} \longrightarrow \partial_{ \pm} \theta_{2}+\varepsilon A_{ \pm}
$$

and the gauge choice $\theta_{2}=0$, combined with the addition of the following piece:

$$
\Delta \mathcal{S}_{D+2}=-\frac{1}{2} \int d^{2} \sigma \varepsilon\left[\partial \theta_{1} \bar{\partial} \theta_{2}-\partial \theta_{2} \bar{\partial} \theta_{1}\right],
$$

which, upon gauging, becomes

$$
-\frac{1}{2} \int d^{2} \sigma\left[\bar{A} \partial \theta_{1}-A \bar{\partial} \theta_{1}\right]
$$


this ensures the invariance of the action (2.1) under finite gauge transformations. After the gauge fixing, the quotient action has the following form (we have dropped the subscript of $\theta_{1}$ ):

$$
\begin{aligned}
\mathcal{S}_{q}[\varepsilon, \theta, x]= & -\frac{1}{2} \int d^{2} \sigma\left[\frac{1-\varepsilon B}{1+\varepsilon B} \partial \theta \bar{\partial} \theta-\frac{\varepsilon F_{2 j}}{1+\varepsilon B} \partial \theta \bar{\partial} x^{j}\right. \\
& +\frac{F_{i 1}}{1+\varepsilon B} \partial x^{i} \bar{\partial} \theta+\left(F_{i j}-\frac{\varepsilon}{2} \frac{F_{i 1} F_{2 j}}{1+\varepsilon B}\right) \partial x^{i} \bar{\partial} x^{j} \\
& \left.-\frac{\sqrt{\gamma}}{4} \mathbf{R}^{(2)}(\mathbf{\Phi}+\ln \operatorname{det}(1+\varepsilon B))\right],
\end{aligned}
$$

where the correction to the dilaton field $\mathbf{\Phi}(x)$ was acquired while integrating out the gauge fields $A_{ \pm}$by their equations of motion and it is equal to the corresponding Jacobian. It is easy to see that the two quotients corresponding to $\varepsilon=1$ and $\varepsilon=-1$ are T-dual to each other. The above result can be straightforwardly generalized for the case of $d$ abelian isometries yielding a family of mutually dual models [6].

As we have just seen, in the case of abelian isometries, the notions of duality and quotient are closely related: a dual pair of $\sigma$-models can be constructed from a higher-dimensional $\sigma$-model; and, conversely, any two axial-vector quotients are related by a suitable duality transformation.

In this article we intend to give a geometric characterization for the nonabelian case. We will show how, given an abstract $\sigma$-model, one can determine if there exists a dual to it that can be obtained by the quotient procedure.

\section{Generalized Non-Abelian Duality}

In this section we introduce the formalism of generalized non-abelian duality (GNAD) proposed by Klimčik and Ševera [1]. The "traditional" non-abelian

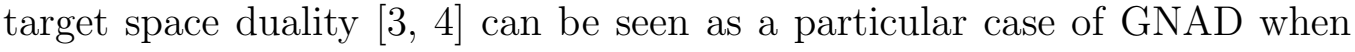
the "dual group" (cf. (3.4),(3.5)) happens to be abelian.

The central idea of [1] was to abandon the requirement for the $\sigma$-model to have a set of isometries (and corresponding to them conserved currents) in favor of so-called "non-commutative conservation laws". This is a weaker property that can be found in the dual theories.

Suppose we are given some $\sigma$-model (2.2) defined on a target manifold $\mathcal{M}$ and we vary the coordinates on $\mathcal{M}$ using the action of some Lie group 
$\mathcal{G}: \delta x^{i}=\varepsilon^{a} v_{a}^{i}$, where $v^{i}{ }_{a}\left(x^{i}\right)$ is a (left) invariant frame field on the group manifold. We may definet the Lie derivatives of the background fields and the currents equal to

$$
\begin{aligned}
& \mathcal{L}_{v_{a}}\left(F_{i j}\right)=F_{i j, k} v_{a}^{k}+F_{k j} v_{a, i}^{k}+F_{i k} v_{a, j}^{k}, \quad \mathcal{L}_{v_{a}}\left(\boldsymbol{\Phi}\left(x^{i}\right)\right)=\boldsymbol{\Phi}_{, k} v_{a}^{k} \\
& \bar{J}_{a}=v_{a}^{i} F_{i j} \bar{\partial} x^{j}, \quad J_{a}=v_{a}^{i} F_{j i} \partial x^{j}
\end{aligned}
$$

Then the variation of the action is

$$
\mathcal{S}_{\sigma}\left[x^{i}+\varepsilon^{a} v_{a}^{i}\right]-\mathcal{S}_{\sigma}\left[x^{i}\right]=\int d^{2} z\left[\varepsilon^{a} \mathcal{L}_{v_{a}}(L)+\partial \varepsilon^{a} \bar{J}_{a}+\bar{\partial} \varepsilon^{a} J_{a}\right],
$$

where $\mathcal{L}_{v_{a}}(L)$ schematically denotes the terms coming from (3.1). Note that $\mathcal{G}$ needs not to be an isometry group of $\mathcal{M}$, since that would amount to the much stricter requirement $\mathcal{L}_{v_{a}}(L)=0$ than the one we are going to impose.

We will say that the given model admits "non-commutative conservation laws" if the currents (3.2) can be locally represented (on-shell) as

$$
\bar{J}_{a} d \bar{z}-J_{a} d z=\operatorname{Tr}\left(\tilde{T}_{a} \tilde{g}^{-1} d \tilde{g}\right),
$$

where $\tilde{g} \in \tilde{\mathcal{G}}$ is the "dual quasi-isometry group" or, equivalently, that they obey the Maurer-Cartan equation'.

$$
\partial \bar{J}_{a}+\bar{\partial} J_{a}+\tilde{f}_{a}^{b c} J_{b} \bar{J}_{c}=0,
$$

Obviously, the Lie derivative terms of (3.3) should then satisfy the condition

$$
\mathcal{L}_{v_{a}}(L)+\tilde{f}_{a}^{b c} J_{b} \bar{J}_{c}=0,
$$

or, after substituting the (3.2), equation (3.7) turns into the condition on the target $\mathcal{M}$ :

$$
\mathcal{L}_{v_{a}}\left(F_{i j}\right)+\tilde{f}_{a}^{b c} v_{b}^{k} F_{i k} v_{c}^{l} F_{l j}=0
$$

Note that (3.7) is the central statement of the Klimčik-Ševera approach, because it provides us with a geometrical, non-dynamical equation.

Conversely, if we establish the off-shell validity of (3.7), then (3.4) and (3.5) will follow immediately (on-shell).

\footnotetext{
${ }^{2}$ As we are going to see in the next section, non-abelian axial-vector duality provides us with the $\sigma$-models that violate (3.2). This makes them interestingly distinct from the case considered by Klimčik and Ševera.

${ }^{3}$ Note that the usual conservation law is simply $\partial \bar{J}_{a}+\bar{\partial} J_{a}=0$.
} 
It is easy to see now that the dual $\sigma$-model should obey the same condition as (3.6) but with the tilded and un-tilded variables interchanged:

$$
\begin{aligned}
\tilde{\bar{J}}_{a} d \bar{z}-\tilde{J}_{a} d z & =\operatorname{Tr}\left(T_{a} g^{-1} d g\right), \quad g \in \mathcal{G} \\
\mathcal{L}_{\tilde{v}_{a}}(\tilde{L})+f_{a}^{b c} \tilde{J}_{b} \tilde{\bar{J}}_{c} & =0
\end{aligned}
$$

From the integrability condition on the Lie derivative (3.7), one can easily check that the original and dual structure constants $f$ and $f$ should obey the relation

$$
f_{d c}^{a} \tilde{f}_{a}^{r s}=\tilde{f}_{c}^{a s} f_{d a}^{r}+\tilde{f}_{c}^{r a} f_{d a}^{s}-\tilde{f}_{d}^{a s} f_{c a}^{r}-\tilde{f}_{d}^{r a} f_{c a}^{s},
$$

which is known in mathematics to be the relation for the structure constants of the Lie bi-algebra $(\mathcal{G}, \tilde{\mathcal{G}})[\mathbb{1}]$.

\section{Non-Abelian Duality and Quotients}

This time we consider the most general action that has non-abelian $\mathcal{G}_{L} \otimes \mathcal{G}_{R}$ (anti)chiral symmetry [2]:

$$
\mathcal{S}_{D+2 d}\left[h_{i}, x\right]=I\left[h_{1}\right]+I\left[h_{2}\right]+\int d^{2} z \operatorname{Tr}\left[\bar{J}_{2} B(x) J_{1}+\bar{J}_{2} \mathcal{F}^{2}+\overline{\mathcal{F}}^{1} J_{1}\right]+\mathcal{S}_{\mathcal{X}},
$$

where $\mathcal{S}_{\mathcal{X}}$ is the same as in (2.2) and the normalization of the WZW actions is fixed by the Polyakov-Wiegmann formula

$$
I\left[h_{1} h_{2}\right]=I\left[h_{1}\right]+I\left[h_{2}\right]+\int d^{2} z \operatorname{Tr}\left[\bar{J}_{2} J_{1}\right]
$$

We have defined

$$
\begin{aligned}
J_{i} & =h_{i}^{-1} \partial h_{i} & \bar{J}_{i}=\bar{\partial} h_{i} h_{i}^{-1} \\
\operatorname{Tr}\left(\overline{\mathcal{F}}^{1} T_{a}\right) & =\bar{\partial} x^{i} F_{i a}^{1}(x) & \operatorname{Tr}\left(\mathcal{F}^{2} T_{a}\right)=F_{a i}^{2}(x) \partial x^{i}
\end{aligned}
$$

and omitted the unnecessary factors in front of the integrals. The resulting chiral currents can be found to be

$$
J_{R}=J_{2}+\omega^{2}\left(B J_{1}+\mathcal{F}^{2}\right) \quad \bar{J}_{L}=\bar{J}_{1}+\left(\bar{J}_{2} B+\overline{\mathcal{F}}^{1}\right) \omega^{1},
$$

\footnotetext{
${ }^{4}$ It can be shown that if one tries to use the Principal Chiral Model actions instead of the WZW actions in (4.1), the resulting quotient $\sigma$-models do not reduce correctly to the abelian case (2.6).
} 
where $\omega_{a b}^{i}=\operatorname{Tr}\left(h_{i} T_{a} h_{i}^{-1} T_{b}\right)$.

Following [2], we note the chiral symmetries of (4.1)

$$
h_{1} \longrightarrow u^{-1} h_{1} \quad \text { and } \quad h_{2} \longrightarrow h_{2} w^{-1}
$$

and that we can gauge them with $A=J\left[h_{L}\right]$ and $\bar{A}=\bar{J}\left[h_{R}\right]$, provided the fields transforms as

$$
h_{1} \longrightarrow u^{-1} h_{1}, \quad h_{L} \longrightarrow h_{L} u ; \quad h_{2} \longrightarrow h_{2} w^{-1}, \quad h_{R} \longrightarrow w h_{R},
$$

where we want $u$ and $w$ to be related by an outer automorphism of the group $\mathcal{G}: \hat{s}\left[w_{\bullet}\right]=u^{-1} \hat{s}[\bullet]$. An outer automorphism is a transformation of a Lie group onto itself by something that is not its own element.

The gauged action is

$$
\begin{aligned}
\mathcal{S}_{D+2 d, \text { gauged }}= & I\left[h_{L} h_{1}\right]+I\left[h_{2} h_{R}\right]-I\left[h_{L} \hat{s}\left[h_{R}\right]\right]+ \\
& \int d^{2} z \operatorname{Tr}\left[\bar{J}\left[h_{2} h_{R}\right] B(x) J\left[h_{L} h_{1}\right]\right. \\
+ & \left.\bar{J}\left[h_{2} h_{R}\right] \mathcal{F}^{2}+\overline{\mathcal{F}}^{1} J\left[h_{L} h_{1}\right]\right]+\mathcal{S}_{\mathcal{X}}
\end{aligned}
$$

We can parametrize the outer automorphism $\hat{s}$ in the chosen representation by the matrix $S$ :

$$
I\left[h_{L} \hat{s}\left[h_{R}\right]\right]=I\left[h_{L}\right]+I\left[h_{R}\right]+\int d^{2} z \operatorname{Tr}\left[\bar{J}\left[h_{R}\right] S J\left[h_{L}\right]\right],
$$

and rewrite the (4.8) as

$$
\mathcal{S}_{D+2 d, \text { gauged }}=\mathcal{S}_{D+2 d}+\int d^{2} z \operatorname{Tr}\left[\bar{A}\left(\omega^{2} B \omega^{1}-S\right) A+\bar{A} J_{R}+\bar{J}_{L} A\right]
$$

The quotient action obtained as in Section 2 (in the gauge $h_{2}=1$ ) is given by (note that $\left.\left(\bar{\partial} h h^{-1}\right)_{a}=\left(h^{-1} \bar{\partial} h\right)_{b} \omega_{b a}^{h}\right)$ :

$$
\mathcal{S}_{q}[S, h, x]=I[h]+\int d^{2} z \operatorname{Tr}\left[\overline{\mathcal{F}}^{1} \omega^{h} j+\overline{\mathcal{I}} M \mathcal{I}\right]+\mathcal{S}_{\mathcal{X}}[x, \Phi-\ln \operatorname{det} M],
$$

where we have defined

$$
M=\left(B^{-1} S\left(\omega^{h}\right)^{-1}-\mathbf{1}\right)^{-1}, \quad j_{ \pm}=\partial_{ \pm} h h^{-1}
$$

and

$$
\overline{\mathcal{I}}=\bar{j} \omega^{h^{-1}}+\overline{\mathcal{F}}^{1}, \quad \mathcal{I}=\omega^{h} j+B(x)^{-1} \mathcal{F}^{2}
$$


The action of the infinitesimal $g=e^{\varepsilon^{a} T_{a}} \in \mathcal{G}$ on the right, $h \longrightarrow h g$, translates into the same perturbation as used in $(3.3)$, because $\left(\mathcal{L}_{x} \bullet \equiv[x, \bullet]\right)$

$$
\begin{aligned}
e^{x} e^{\varepsilon} & =\exp \left(x-\frac{\mathcal{L}_{x}}{e^{-\mathcal{L}_{x}}-1} \varepsilon\right), \\
h^{-1} d h & \equiv T_{a}\left(v^{i}{ }_{a}\right)^{-1} d x^{i}=\left(e^{-\mathcal{L}_{x}}-1\right) d=\left(e^{-\mathcal{L}_{x}}-1\right) \frac{1}{-\mathcal{L}_{x}} d x
\end{aligned}
$$

We obtain $\left((\varepsilon f)_{b c} \equiv-\varepsilon^{a} f_{a b c}\right)$

$$
\begin{aligned}
\omega^{g} & =1-(\varepsilon f) \\
\delta j_{ \pm} & =\partial_{ \pm} \varepsilon \omega^{h}=\omega^{h^{-1}} \partial_{ \pm} \varepsilon \\
\delta \omega^{h} & =-(\varepsilon f) \omega^{h}, \quad \delta \omega^{h^{-1}}=\omega^{h^{-1}}(\varepsilon f) \\
\delta M & =-(1+M)(\varepsilon f) M \\
\delta \mathcal{I} & =\partial \varepsilon-(\varepsilon f) \omega^{h} j, \quad \delta \overline{\mathcal{I}}=\bar{\partial} \varepsilon+\bar{j} \omega^{h^{-1}}(\varepsilon f)
\end{aligned}
$$

And the resulting variation is

$$
\delta_{g} \mathcal{S}_{q}[h, x]=\int d^{2} z \operatorname{Tr}\left[\partial \varepsilon \bar{J}_{q}+\bar{\partial} \varepsilon J_{q}\right]-\int d^{2} z \varepsilon^{a} f_{a}^{b c} J_{q}^{b} \bar{J}_{q}^{c},
$$

where the "non-commutative currents" introduced in (3.5) are explicitly given by

$$
J_{q}=\omega^{h} j+M \mathcal{I} \quad \bar{J}_{q}=\overline{\mathcal{F}}^{1}+\overline{\mathcal{I}} M .
$$

Here we have to stop and observe two facts: 1) the above $J_{ \pm q}$ are not given by $(3.2) ; 2)(3.10)$ is not obeyed for $\tilde{\mathcal{G}}=\mathcal{G}$, unless we want them to be Borelian.

The contradiction can be resolved if we notice that the quotient currents (4.22) can be written as:

$$
\begin{aligned}
J_{q a} & =v_{a}^{i}\left(F_{i j}+F_{i j}^{W Z W(-)}\right) \partial x^{j} \\
\bar{J}_{q a} & =\bar{\partial} x^{i}\left(F_{i j}-F_{i j}^{W Z W(+)}\right) v_{a}^{j}
\end{aligned}
$$

where $F_{i j}^{W Z W( \pm)}$ are the WZW backgrounds with different signs of the WZ term.

Then we find

$$
\begin{aligned}
\mathcal{L}_{v_{a}}\left(F_{i j}\right) & =\tilde{f}_{a}^{b c}\left(F_{i k}-F_{i k}^{W Z W(+)}\right) v_{b}^{k} v_{c}^{l}\left(F_{l j}+F_{l j}^{W Z W(-)}\right) \\
\mathcal{L}_{v_{a}}\left(F_{i j}^{W Z W( \pm)}\right) & =0
\end{aligned}
$$

\footnotetext{
${ }^{5}$ We thank Ctirad Klimčik for this information.
} 
Equation (4.25) is our main result. It is the NAAVD analog of the Killing equation for abelian duality and of (3.7) for the Klimčik-Ševera duality.

Our integrability condition acquires an extra term on the right hand side when compared to $(3.10)$ :

$$
f_{d c}^{a} \tilde{f}_{a}^{r s}=\tilde{f}_{c}^{a s} f_{d a}^{r}+\tilde{f}_{c}^{r a} f_{d a}^{s}-\tilde{f}_{d}^{a s} f_{c a}^{r}-\tilde{f}_{d}^{r a} f_{c a}^{s}-\tilde{f}_{d c}^{a} \tilde{f}_{a}^{r s}
$$

It comes from the part $]$ of $\left[\mathcal{L}_{v_{c}}, \mathcal{L}_{v_{d}}\right]\left(F_{i j}\right)$ cubic in background fields, where we have used the fact that

$$
\left(F+F^{W Z W(-)}\right)_{i j}-\left(F-F^{W Z W(+)}\right)_{i j}=\mathbf{1}
$$

This term is vital for our scheme, because for $\tilde{f}=f$ (4.27) becomes simply a Jacobi identityl.

Thus we have completed the circle of transformations for the non-abelian axial-vector duality. We know how to construct a pair of dual models from a base model, and how (using the equation (4.25) ) to determine if a given $\sigma$-model (in the absence of obvious isometries) can be dualized.

The one parameter family of theories (4.11) has the duality group $\operatorname{Aut}(\mathcal{G})$, which is the group of outer automorphisms of $\mathcal{G}$, as the automorphism parameter matrix $S$ enters into $M$.

\section{Conclusions}

Non-abelian axial-vector duality provides a useful tool to gain insight into the rich geometric structure of duality which has been hidden for some time by the simplicity of abelian systems. Right now there are two procedures: 1) axial-vector, which has a clear path-integral interpretation; 2) bi-algebra, where we have a clear classification, but no underlying path-integral mechanism.

The old $\int \operatorname{Tr}(\lambda F)$ mechanism [8] turns out to be inadequate for this more complex environment; and, should the target space duality be a relevant symmetry of the string theory, we need to find a unified description for it.

The other problem to be solved for Kiritsis-Obers duality is the conformal structure of the dual models. As we know, $U(1)$ axial-vector quotients are identical from the CFT point of view, and one needs to find out if this situation persists for non-abelian groups.

\footnotetext{
${ }^{6}$ which is identically zero for GNAD

7 We have formally introduced $\tilde{f}$ in $(4.25),(4.27)$ just to make a comparison with [1].
} 


\section{Acknowledgments}

It is a great pleasure to thank Martin Roček for suggesting this problem to me and for his guidance and advice. I am also grateful to Dileep Jatkar and Ctirad Klimčik for stimulating discussions.

\section{References}

[1] C. Klimčik, P. Ševera "Dual Non-Abelian Duality and the Drinfeld Double", hep-th/9502122

[2] E. Kiritsis, N. Obers "A New Duality Symmetry in String Theory", Phys. Lett. B334 (1994) 67 or hep-th/9406082

[3] A. Giveon, M. Roček "On Nonabelian Duality", Nucl. Phys. B421 (1994) 173 or hep-th/9308154

[4] F. Quevedo "Abelian and Non-Abelian Dualities in String Backgrounds", hep-th/9305055

X. de la Ossa, F. Quevedo "Duality Symmetries from Non-Abelian Isometries in String Theory", Nucl. Phys. B403 (1993) 377 or hep-th/9210021

[5] K. Sfetsos "Gauged WZW Models and Non-Abelian Duality", Phys. Rev. D50 (1994) 2784 or hep-th/9402031

[6] A. Giveon, M. Roček "Generalized Duality in Curved StringBackgrounds", Nucl. Phys. B380 (1992) 128 or hep-th/9112070

[7] M. Roček , E. Verlinde "Duality, Quotients and Currents", Nucl. Phys. B373 (1992) 630 or hep-th/9110053

[8] T. Buscher "Studies of the Two-dimensional Nonlinear Sigma-model", Ph. D. thesis (1988), unpublished

T. Buscher "Path-Integral Derivation of Quantum Duality In Nonlinear Sigma-Models", Phys. Lett. B201 (1988) 466

T. Buscher "A Symmetry of the String Background Field Equations", Phys. Lett. B194 (1987) 59 
[9] E. Álvarez, L. Álvarez-Gaumè, Y. Lozano "An Introduction to T-Duality in String Theory", hep-th/9410327

[10] Y. Lozano "Non-Abelian Duality and Canonical Transformations", hep-th/9503045 